\title{
Frequency of Pediatric Acute Respiratory Distress Syndrome Based on Oxygen Saturation Index in Pediatric Intensive Care Unit of a Developing Country
}

\author{
Rahim Ahmed ${ }^{1}$, Asim Azim ${ }^{1}$, Azam Nangialay ${ }^{2}$, Anwar Haque ${ }^{3}$, Humaira Jurair ${ }^{4}$
}

1. Pediatric Intensive Care Unit (PICU), The Indus Hospital, Karachi, PAK 2. Pediatrics, Aga Khan University Hospital, Karachi, PAK 3. Pediatrics, The Indus Hospital, Karachi, PAK 4. Pediatric Intensive Care Unit (PICU), Aga Khan University Hospital, Karachi, PAK

Corresponding author: Rahim Ahmed, rahim.ahmed1983@gmail.com

\section{Abstract \\ Objectives}

To determine the frequency of pediatric acute respiratory distress syndrome based on oxygen saturation index in pediatric intensive care unit of a developing country.

\section{Methods}

We conducted a retrospective study of all children admitted in pediatric intensive care unit (PICU) of Aga Khan University Hospital, Karachi from July 2017 to June 2018 with respiratory rate >40 breaths/minute, shortness of breath, and bluish discoloration of skin and mucous membranes. The diagnosis of acute respiratory distress syndrome (ARDS) was made on the basis of standard operational definitions as mentioned (fulfilling criteria for ARDS).

\section{Results}

During the one-year study period 150 patients with age range of one month to 16 years were admitted fulfilling the inclusion criteria. Mean age was $38.27 \pm 53.13$ months, and 92 (61.33\%) were male with male to female ratio of 1.6:1. Mean duration of symptoms was $1.23 \pm 0.42$ days. Frequency of pediatric acute respiratory distress syndrome using oxygen saturation index admitted in a pediatric ICU was 23 (15.33\%) patients.

\section{Conclusion}

This study has shown that the frequency of pediatric acute respiratory distress syndrome is quite high.

Received 12/09/2019

Review began 12/17/2019 Review ended 12/18/2019 Published 12/22/2019

๑) Copyright 2019

Ahmed et al. This is an open access article distributed under the terms of the Creative Commons Attribution License CC-BY 3.0., which permits unrestricted use, distribution, and reproduction in any medium, provided the original author and source are credited.
Categories: Pediatrics

Keywords: pediatric acute respiratory distress syndrome, oxygen saturation index, picu

\section{Introduction}

Acute respiratory distress syndrome (ARDS), evident by persistent hypoxemia, decrease respiratory system compliance and nonhydrostatic pulmonary edema, is a serious and complex clinical problem with a high morbidity, mortality and financial cost, especially in a resource-limited situation. The reported morbidity and mortality ranges from $14 \%$ to $61 \%$ in developed countries and even high in developing countries [1,2]. It involves a series of events following acute lung injury and can be triggered by a variety of insults, including pneumonia, sepsis, aspiration, shock, burns and traumatic injury, all results in inflammation and increased vascular permeability leading to pulmonary edema [3].

ARDS was defined in different times and due to lack of universal accepted definition till now has led to a series of practical problems for a definitive diagnostic guidelines and hence therapeutic strategies. ARDS was previously diagnosed on the basis of American-European consensus conference definition, changed to Berlin definition in 2012, by European intensive medicine society [4,5]. Recently, the pediatric acute lung injury consensus conference group described the latest definition of pARDS based on oxygen saturation (OI) and oxygen saturation index (OSI) [6].

It is not uncommon diagnosis in pediatrics intensive care units (PICUs) and estimated prevalence ranges from $1.4 \%$ to $2.7 \%$ of all PICU's admissions [1,3]. The reported data regarding incidence of pARDS varies from study to study and time to time depending on the patient population targeted. The epidemiological data available is mostly from the developed countries and there is scant data on incidence of pARDS in developing countries [3,7]. A recent study from India reported pARDS incidence from 8.5 to 27 cases per 
1000 PICU admissions [2]. Another study from Iran reports incidence from 5 to $10 \%$ of total PICU admissions [8]. We found no available data on incidence of pARDS in Pakistan after a thorough literature search.

Pulse oximetry is the most commonly utilized technique to monitor oxygenation, noninvasive, and safe. Pulse oximetry prevents arterial blood sampling and curtails cost for arterial blood gas analysis [9]. The oxygenation saturation index (OSI) and oxygen saturation (SF) ratio used as an oxygenation metrics instead of oxygenation index (OI) [Fraction of inspired oxygen concentration x mean airway pressure / pulse oximetry saturation] or PF ratio [partial pressure of oxygen/fraction of inspired oxygen concentration] will lead to earlier diagnosis and initiation of treatment in pediatric acute respiratory distress syndrome especially in resource-limited country [10].

\section{Rationale}

In previous studies done, the criteria used to label pediatric acute respiratory distress syndrome (pARDS) was based on invasive procedure for assessment of oxygenation. Nowadays, OSI is used as a component to define pARDS, which is non-invasive and readily available. By using this criteria we aim to assess magnitude of pARDS in children admitted to pediatric intensive care unit.

\section{Materials And Methods}

This was a hospital-based descriptive study conducted at the PICU of Aga Khan University Hospital over a period of one year from July 2017 to June 2018 after the approval from the Institutional Review Board. By using WHO calculator taking prevalence of pediatric acute respiratory distress syndrome as $66.4 \%$ margin of error equal to $7.6 \%$, the calculated sample size will be as 150 [3].

\section{Operational definitions}

OSI: Fraction of inspired oxygen concentration x mean airway pressure/ pulse oximetry saturation

ARDS: Acute onset (within seven days of onset) of symptoms like respiratory rate $>40$, bluish discoloration of skin and mucous membranes and shortness of breath with bilateral infiltrates on chest radiography consistent with acute parenchymal disease, presence of generalized edema and oxygen saturation index > 5.0, assisted by probe attaching to finger and shown on monitor and oxygen via face mask or nasal cannula $[6]$.

The demographic data collected included age, gender, weight of child and oxygenation status at the time of admission. Patient was examined for shortness of breath, bluish discoloration of skin and mucous membranes. Respiratory rate was measured by counting the number of breaths per minute. History of onset of symptoms was asked from parents and guardians. X-ray chest, anterior posterior view was done and reviewed by radiologist for presence of infiltrates. Probe was attached, monitor was connected and oxygen support was given via face mask or nasal cannula and OSI was measured.

By using SPSS version 22 (IBM Corp, Armonk, NY) data was analyzed. Mean \pm standard deviation was calculated for age, weight of child, OSI, duration of symptoms. Frequency and percentages were calculated for gender, bilateral chest infiltrates and edema.

Effect modifiers like age, gender, duration of symptoms, weight of child, bluish discoloration of skin and mucous membranes and OSI $>5$ were controlled through stratification. Post stratification chi-square test was applied. P-valve $\leqslant 0.05$ was taken as significant.

\section{Results}

Age range in this study was from one month to 16 years with mean age of $38.27 \pm 53.13$ months. Out of the 150 patients, 92 (61.33\%) were males and 58 (38.67\%) were females with male to female ratio of 1.6:1. Mean duration of symptoms was $1.23 \pm 0.42$ days and mean weight of children was $14.53 \pm 16.64 \mathrm{~kg}$. Distribution of patients according to bilateral chest infiltrates, edema, OSI $>5$ and bluish discoloration of skin and mucous membranes is shown in Figure 1. 


\section{Cureus}

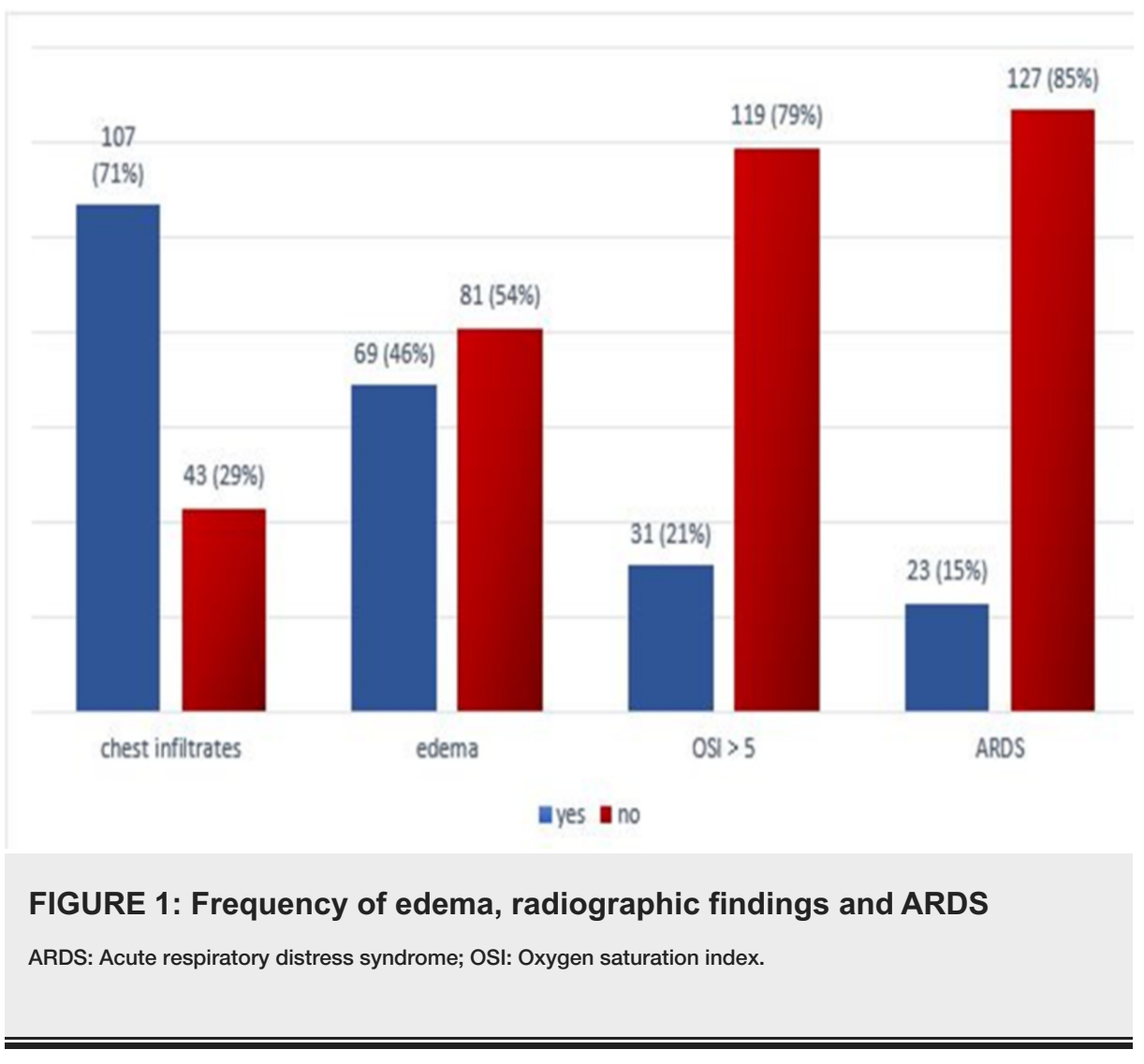

In this study, I have found the frequency of pediatric acute respiratory distress syndrome as per new definition in children admitted in a pediatric ICU in 23 (15.33\%) patients (Figure 1).

Stratification of pediatric acute respiratory distress syndrome with respect to age and gender along with duration of symptoms and weight of baby is shown in Figure 2, respectively. Stratification of pediatric acute respiratory distress syndrome with respect to bilateral chest infiltrates, edema, OSI > 5 and bluish discoloration of skin and mucous membranes is also shown in Figure 2.

\section{- AROSYES IARDSNO}

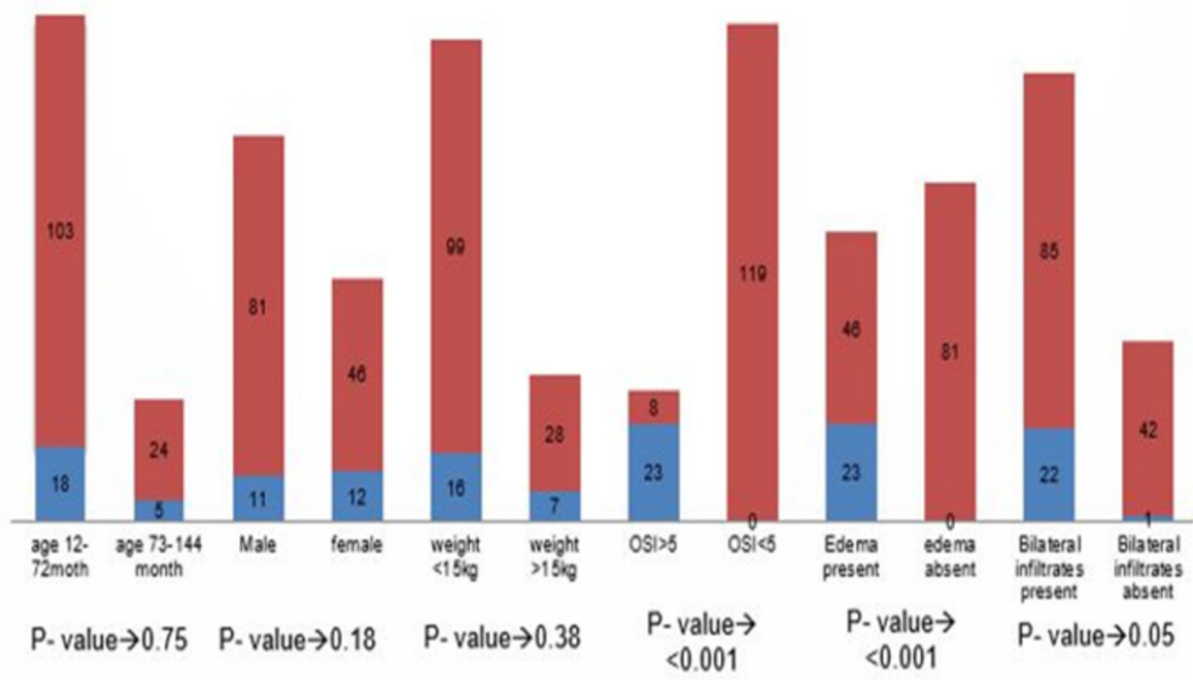

FIGURE 2: Stratification of pARDS with respect to age, gender, weight, OSI, edema and radiological findings

pARDS: Pediatric acute respiratory distress syndrome; OSI: Oxygen saturation index. 


\section{Discussion}

Although the epidemiology of ARDS has been well documented for adults, few epidemiological studies have been conducted with children. Studies that used the American-European Conference Consensus (AECC) definition have shown prevalence from $0.86 \%$ to $7.8 \%$ of PICU admissions, and $5 \%$ to $20.5 \%$ of ventilated patients [11-14]. Mortality rates ranged from $14 \%$ to $61 \%[4,14]$. Higher mortality rates have been reported in developing countries $[11,15]$. Despite the fact that the data used in the Berlin study did not include pediatric patients, its validity for infants and toddlers has recently been demonstrated in a study conducted by the European Society for Pediatric and Neonatal Intensive Care [14]. I have conducted this study to determine the frequency of pediatric acute respiratory distress syndrome as per new definition in children admitted in a pediatric ICU of a tertiary-care university hospital.

Age range in this study was from one month to 16 years with mean age of $38.27 \pm 53.13$ months. Out of the 150 patients, 92 (61.33\%) were males and 58 (38.67\%) were females with male to female ratio of 1.6:1. In this study, I have found the frequency of pediatric acute respiratory distress syndrome as per new definition in children admitted in a pediatric ICU in 23 (15.33\%) patients. A recent study from India reported pARDS incidence from 8.5 to 27 cases per 1000 PICU admissions [2]. Another study from Iran reports incidence from 5 to $10 \%$ of total PICU admissions [8].

The incidence of pediatric ARDS is different than adult and it is relatively rare but it is also underdiagnosed due to lack of specific guidelines. Its prevalence in children in the United States, Europe and Australia is 212.8 cases $/ 100,000$ people per year [16]. In North America, multi-center study reported that $1-4 \%$ of children undergoing mechanical ventilation had ARDS among children hospitalized in PICU [17].

A research by Zimmerman et al. was conducted as a population-based prospective cohort study that was designed to determine the population incidence and outcome of pediatric acute lung injury (ALI) [15]. The research was performed at all hospitals admitting critically-ill children in King County Washington. It was done on children aged six months to 15 years who required invasive (through endotracheal tube or tracheostomy) or non-invasive (through a full-face mask) mechanical ventilation. They utilized the AECC criteria. They found a prevalence of $27.6 \%$ (39/141) for both ALI and ARDS. Furthermore, ALI accounted for $7 \%$ (10/141) while ARDS accounted for $20.6 \%$.

Quartin et al. conducted a prospective cross-sectional study on the prevalence of ALI and ARDS outside the intensive care units [18]. This group evaluated all the adult patients (above 18 years) who were admitted to respiratory isolation rooms on the general wards of a large tertiary hospital over a period of one year. This group utilized the AECC criteria to diagnose the patients. A total of 715 patients were screened, 62 of which fulfilled the ALI screening criteria with a prevalence of $9 \%$. Further, 15 out of 715 fulfilled the ARDS criteria giving a prevalence of $2 \%$.

In comparison, Hughes et al. conducted a prospective cross-sectional study on the prevalence of ARDS in 23 adult intensive care units in Scotland over a period of eight months [19]. A total of 4530 patients aged 15 years and above were recruited into the study. The patients with ARDS were identified using the diagnostic criteria defined by the AECC. About 367 patients were diagnosed with ARDS, giving a prevalence of $8.1 \%$. This study did not assess for patients with ALI. Research by Rubenfeld et al. was conducted on a prospective cohort study in 21 intensive care units in Washington over a period of one year. The group applied the AECC criteria to diagnose the patients. They evaluated mechanically ventilated patients aged 15-80 years. A total of 4251 patients were enrolled. The prevalence of ALI was $26.2 \%$ (1113 out of 4251) and that of ARDS was $19.5 \%$ (828 out of 4251 ) [20].

This study has shown that the frequency of pediatric acute respiratory distress syndrome as per new definition in children admitted in a pediatric ICU is quite high. So, we recommend that this non-invasive and readily available method of OSI should be used as a routine for early diagnosis and management of pediatric acute respiratory distress syndrome.

\section{Conclusions}

Our study done in PICU shows that by using non-invasive OSI, early diagnosis of pediatric acute respiratory distress can be made and this will help in better management and less mortality.

\section{Additional Information \\ Disclosures}

Human subjects: Consent was obtained by all participants in this study. Aga Khan University issued approval 5031-PED-ERC-17. My study has been granted exemption. Animal subjects: All authors have confirmed that this study did not involve animal subjects or tissue. Conflicts of interest: In compliance with the ICMJE uniform disclosure form, all authors declare the following: Payment/services info: All 
authors have declared that no financial support was received from any organization for the submitted work. Financial relationships: All authors have declared that they have no financial relationships at present or within the previous three years with any organizations that might have an interest in the submitted work.

Other relationships: All authors have declared that there are no other relationships or activities that could appear to have influenced the submitted work.

\section{References}

1. Yu WL, Lu ZJ, Wang Y, et al.: The epidemiology of acute respiratory distress syndrome in pediatric intensive care units in China. Intensive Care Med. 2009, 35:136-143. 10.1007/s00134-008-1254-X

2. Chetan G, Rathisharmila R, Narayanan P, Mahadevan S: Acute respiratory distress syndrome in pediatric intensive care unit. Indian J Pediatr. 2009, 76:1013-1016.

3. Hu X, Qian S, Xu F, et al.: Incidence, management and mortality of acute hypoxemic respiratory failure and acute respiratory distress syndrome from a prospective study of Chinese paediatric intensive care network. Acta Paediatr. 2010, 99:715-721. 10.1111/j.1651-2227.2010.01685.x

4. Bernard GR, Artigas A, Brigham KL, et al.: The American-European Consensus Conference on ARDS. Definitions, mechanisms, relevant outcomes, and clinical trial coordination. Am J Respir Crit Care Med. 1994, 149:818-824. 10.1164/ajrccm.149.3.7509706

5. Ferguson ND, Fan E, Camporota L, et al.: The Berlin definition of ARDS: an expanded rationale, justification, and supplementary material. Intensive Care Med. 2012, 38:1573-1582. 10.1007/s00134-0122682-1

6. The Pediatric Acute Lung Injury Consensus Conference Group: Pediatric acute respiratory distress syndrome: consensus recommendations from the pediatric acute lung injury consensus conference. Pediatr Crit Care Med. 2015, 16:428-439. 10.1097/PCC.0000000000000350

7. Villar J, Blanco J, Anon JM, et al.: The ALIEN study: incidence and outcome of acute respiratory distress syndrome in the era of lung protective ventilation. Intensive Care Med. 2011, 37:1932-1941. 10.1007/s00134-011-2380-4

8. Bilan N, Dastranji A, Behbahani AG: Comparison of the Spo2/Fio2 ratio and the Pao2/Fio2 ratio in patients with acute lung injury or acute respiratory distress syndrome. J Cardiovasc Thorac Res. 2015, 7:28-31. 10.15171/jcvtr.2014.06

9. Fouzas S, Priftis KN, Anthracopoulos MB: Pulse oximetry in pediatric practice. Pediatrics. 2011, 128:740752. 10.1542/peds.2011-0271

10. Thomas NJ, Shaffer ML, Willson DF, Shih MC, Curley M: Defining acute lung disease in children with the oxygenation saturation index. Pediatr Crit Care Med. 2010, 11:12-17. 10.1097/PCC.0b013e3181b0653d

11. Li Y, Wang Q, Chen H, Gao HM, Zhou T, Qian SY: Epidemiological features and risk factor analysis of children with acute lung injury. World J Pediatr. 2012, 8:43-46. 10.1007/s12519-012-0334-8

12. Erickson S, Schibler A, Numa A, et al.: Acute lung injury in pediatric intensive care in Australia and New Zealand: a prospective, multicenter, observational study. Pediatr Crit Care Med. 2007, 8:317-323. 10.1097/01.PCC.0000269408.64179.FF

13. Bindl L, Dresbach K, Lentze MJ: Incidence of acute respiratory distress syndrome in German children and adolescents: a population-based study. Crit Care Med. 2005, 33:209-312. 10.1097/01.CCM.0000151137.76768.08

14. De Luca D, Piastra M, Chidini G, et al.: The use of the Berlin definition for acute respiratory distress syndrome during infancy and early childhood: multicenter evaluation and expert consensus. Intensive Care Med. 2013, 39:2083-2091. 10.1007/s00134-013-3110-x

15. Zimmerman JJ, Akhtar SR, Caldwell E, Rubenfeld GD: Incidence and outcomes of pediatric acute lung injury . Pediatrics. 2009, 124:87-95. 10.1542/peds.2007-2462

16. Rotta AT, Piva JP, Andreolio C, de Carvalho WB, Garcia PCR: Progress and perspectives in pediatric acute respiratory distress syndrome. Rev Bras Ter Intensiva. 2015, 27:266-273. 10.5935/0103-507X.20150035

17. Farias JA, Frutos F, Esteban A, et al.: What is the daily practice of mechanical ventilation in pediatric intensive care units? A multicenter study. Intensive Care Med. 2004, 30:918-925. 10.1007/s00134-004-22255

18. Quartin AA, Campos MA, Maldonado D, Ashkin D, Cely CM, Schein RMH: Acute lung injury outside of the ICU. Chest. 2009, 135:261-268. 10.1378/chest.08-0280

19. Hughes M, MacKirdy FN, Ross J, Norrie J, Grant IS; Scottish Intensive Care Society: Acute respiratory distress syndrome; an audit of incidence and outcome in Scottish intensive care units. Anaesthesia. 2003, 58:838-845. 10.1046/j.1365-2044.2003.03287.x

20. Rubenfeld GD, Caldwell PN, Peabody E, et al.: Incidence and outcomes of acute lung injury . N Engl J Med. 2005, 353:1685-1693. 10.1056/NEJMoa050333 